\title{
Reflets
}

Revue d'intervention sociale et communautaire

\section{Niveaux et raisons du sans-abrisme chez les Francophones dans le nord de l'Ontario}

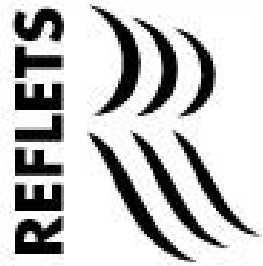

\section{Carol Kauppi, Henri Pallard, Suzanne Lemieux et Thomas Matukala Nkosi}

Volume 18, numéro 1, printemps 2012

L'intervention communautaire chez les francophones minoritaires : des pratiques à découvrir

URI : https://id.erudit.org/iderudit/1012333ar

DOI : https://doi.org/10.7202/1012333ar

Aller au sommaire du numéro

Éditeur(s)

Reflets, Revue d'intervention sociale et communautaire

ISSN

1203-4576 (imprimé)

1712-8498 (numérique)

Découvrir la revue

Citer cet article

Kauppi, C., Pallard, H., Lemieux, S. \& Matukala Nkosi, T. (2012). Niveaux et raisons du sans-abrisme chez les Francophones dans le nord de l'Ontario. Reflets, 18(1), 91-118. https://doi.org/10.7202/1012333ar
Résumé de l'article

La prévalence du sans-abrisme et ses conséquences sociales, économiques, culturelles, ainsi que celles sur le plan de la santé constituent toujours un problème pour les gouvernants et les intervenants dans ce domaine. Cependant, il demeure difficile de définir le sans-abrisme, de dénombrer les sans-abri ou díestimer leur population et de déterminer la méthode appropriée pour étudier le sans-abrisme. En utilisant les données du projet Pauvreté, sans-abrisme et migration (2011), nous avons étudié les niveaux, expériences et raisons du sans-abrisme chez les Francophones à Timmins et à North Bay et nous les avons comparés à ceux des Anglophones et des Autochtones de ces deux communautés.
Tous droits réservés @ Reflets, Revue d’intervention sociale et communautaire, 2012
Ce document est protégé par la loi sur le droit d’auteur. L'utilisation des services d'Érudit (y compris la reproduction) est assujettie à sa politique d'utilisation que vous pouvez consulter en ligne. 


\section{Niveaux et raisons du sans- abrisme chez les Francophones dans le nord de l'Ontario}

Carol Kauppi, "Pauvreté, sans-abrisme et migration ", Centre de recherche sur la justice et la politique sociales, Ecole de service social, Université Laurentienne ${ }^{1}$

Henri Pallard, "Pauvreté, sans-abrisme et migration ", Centre de recherche sur la justice et la politique sociales, Département de droit et justice, Université Laurentienne

Suzanne Lemieux, "Pauvreté, sans-abrisme et migration », Centre de recherche sur la justice et la politique sociales, Université Laurentienne

Thomas Matukala Nkosi, "Pauvreté, sans-abrisme et migration ", Centre de recherche sur la justice et la politique sociales, École de service social, Université Laurentienne

\section{Résumé}

La prévalence du sans-abrisme et ses conséquences sociales, économiques, culturelles, ainsi que celles sur le plan de la santé constituent toujours un problème pour les gouvernants et les intervenants dans ce domaine. Cependant, il demeure difficile de définir le sans-abrisme, de dénombrer les sans-abri ou díestimer leur population et de déterminer la méthode appropriée pour étudier le sans-abrisme. En utilisant les données du projet Pauvreté, sans-abrisme et migration (2011), nous avons étudié les niveaux, expériences et raisons du sans-abrisme chez les Francophones à Timmins et à North Bay et nous les avons comparés à ceux des Anglophones et des Autochtones de ces deux communautés. 
Mots clés : sans-abri absolu, à risque de devenir sans-abri, sans-abrisme, prévalence du sans-abrisme, Nord de l'Ontario, Francophones sans-abri, Autochtones sans-abri.

\section{Abstract}

The prevalence of homelessness and its social, economic, cultural, and health consequences are a problem for governments and others working in this field. However, it is difficult to define homelessness, to count homeless persons or to estimate their population, and to determine the appropriate method to be used in a study. Using data from the "Poverty, homelessness and migration" project, the levels, experiences and reasons for homelessness among Francophones in Timmins and North Bay were studied and compared with those of Anglophones and Aboriginals in these two communities.

Key words : absolutely homeless, at risk of homelessness, homelessness, prevalence of homelessness, northeastern Ontario, homeless Francophones, homeless Aboriginals.

\section{Introduction}

En dépit de la bonne réputation du Canada au niveau international pour sa qualité de vie (Frankish, Hwang et Quantz, 2005), les sansabri sont de plus en plus nombreux. Une nuit, on en a dénombré 5000 à Toronto, 900 à Ottawa et 2700 à Vancouver (Hwang, et collab., 2011). Globalement et sur une base annuelle, on estime que le nombre de sans-abri varie entre 150000 et 300000 au Canada (Hwang, et collab., 2011). Le dénombrement des sans-abri au pays a commencé en 1987 via les travaux du Conseil canadien de développement social (Begin, et collab., 1999). Le recensement général de la population canadienne de 2001 (Statistique Canada, 2002) estimait à plus de 14000 le nombre de sans-abri au pays. Mais ce chiffre représente vraisemblablement une sous-estimation (Frankish, Hwang et Quantz, 2005). En effet, « il demeure 
difficile de définir le sans-abrisme, de dénombrer les sans-abri ou díestimer la taille de leur population, et de déterminer la méthode appropriée pour líétudier " (Kauppi, Bélanger et Partridge, 2003, p. 3). Potter et Gauvreau (2005, p. 6) soulignent également quíil "n'est pas facile d'évaluer la population des sans-abri, d'autant plus que la définition de ce terme comprend la population à risque de devenir sans-abri, de même que les personnes qui vivent dans des refuges d'urgence ou dans la rue ». Frankish, Hwang et Quantz, (2005) ont donc proposé le développement de nouvelles stratégies pour mieux orienter la recherche dans la lutte contre le phénomène des sans-abri ou de l'itinérance ${ }^{3}$.

Depuis, plusieurs initiatives ont été prises, comme la mise en place du Système d'information sur les personnes et les familles sans-abri (SISA) et le dénombrement des sans-abri dans certaines villes canadiennes dontVancouver,Toronto, Calgary et Edmonton (Woodward, et collab., 2002;Ville de Toronto, 2000; Stroik, 2004). C'est dans ce contexte que Kauppi, Bélanger et Partridge (2003) ont mené une enquête sur le nombre de sans-abri dans Sudbury en utilisant des techniques fondées sur la prestation de services et décrites par Iachan et Dennis (1993, dans Peressini, McDonald et Hulchanski, 1996). Selon ces techniques, en s'enquérant auprès des fournisseurs de services aux sans-abri, les chercheurs identifient ces derniers et les rencontrent afin d'obtenir des réponses à leurs questions. Cette façon de procéder est répandue à un point tel que depuis la fin des années 1980 la plupart des études menées sur les sans-abri en utilisent une variante (Peressini, McDonald et Hulchanski, 1996).

Selon les chercheurs dans le domaine, plusieurs questions restent en suspens. En utilisant des méthodologies différentes, telles que le dénombrement des personnes "sur vingt-quatre heures " ou de celles "dormant dans la rue ", le nombre obtenu de sansabri n'inclut pas nécessairement des personnes appartenant à des sous-groupes particuliers à cette population. Cependant selon une étude, de toutes les méthodes visant à dénombrer les sansabri, celles fondées sur les services produisent les résultats les plus probants (Peressini, McDonald et Hulchanski, 2010), certainement des données plus fiables que celles du recensement réalisé par 
Statistique Canada. De plus, la collecte de données auprès des fournisseurs de services - refuges, soupes populaires, centres d'accueil, banques alimentaires, services sociaux et services de santé physique et mentale — permet d'identifier jusqu'à $95 \%$ des sansabri vivant en milieu urbain (Peressini, McDonald et Hulchanski, 2010). C'est la raison pour laquelle nous avons privilégié cette méthodologie pour la présente étude.

En outre, comme les techniques fondées sur les services permettent de recueillir pendant sept jours consécutifs des renseignements détaillés sur chaque pensionnaire de refuges ou usager de services, il a été possible de recenser les clients réguliers et ceux qui ne sont que de passage (Kauppi, Bélanger et Partridge, 2003). Le dénombrement des sans-abri par un compte de prévalence au cours d'une période donnée permet une image plus précise de cette population, alors que le fait de les recenser à un moment donné - au cours d'une seule journée - quoiqu'il réduise le temps et l'effort requis pour rassembler les données, produit souvent des chiffres approximatifs. En effet, certaines personnes ne se retrouvent pas nécessairement dans la rue ou ne sollicitent pas les services d'un fournisseur le jour du dénombrement. De plus, en laissant aux fournisseurs le soin d'effectuer le dénombrement, on réduit le risque de violer la confidentialité des clients et de s'ingérer dans les services offerts par les fournisseurs (Kauppi, Bélanger et Partridge, 2003).

Mais au-delà du chiffre brut de sans-abri, il s'avère important de connaître les motifs derrière l'itinérance et les expériences des personnes qui la vivent (Kauppi, Bélanger et Partridge, 2003). Il faut reconnaitre que plusieurs travaux ont pu faire avancer les connaissances sur la situation des personnes sans-abri au Canada; il n'en reste pas moins qu'aucun d'eux ne s'est penché sur l'expérience de l'itinérance vécue par des personnes appartenant à des groupes minoritaires. D'une part, l'itinérance se pose différemment d'une province à l'autre du pays et, dans une même province, elle touche différemment les catégories sociales de la population. D'autre part, si les personnes minoritaires font face plus que d'autres à des difficultés d'accès au marché de l'emploi, à l'éducation et aux ressources sociales existantes (Picard, 2000; 
"Il s'agit donc de savoir si le fait d'appartenir à une composante donnée de la population, en l'occurrence la composante francophone du Nord de l'Ontario, vivant en situation minoritaire, peut expliquer en partie le statut de sans-abri absolu ou de personne à risque de le devenir."
Picard, et Allaire, 2005; Bouchard, et collab., 2009), on peut penser qu'elles peuvent être plus touchées par le phénomène de l'itinérance.

S'appuyant sur les données collectées par Kauppi et son équipe en 2011, cet article se propose justement de répondre à cette problématique en ce qui concerne la population francophone du Nord de l'Ontario. Nous nous demandons si cette dernière, qui est minoritaire, est plus touchée par l'itinérance que les populations anglophones ou autochtones du nord de la province. Plus précisément, la présente étude fait état du compte de sansabri francophones dans les villes de Timmins et de North Bay. Nous comparerons également ces données à celles déjà recueillies sur les sans-abri francophones de Sudbury. En même temps, cette étude vérifiera si l'expérience de l'itinérance des Francophones differe de celle des autres groupes de la population à l'étude les Anglophones et les Autochtones - et si dans l'ensemble les Francophones présentent des caractéristiques différentes au chapitre du sans-abrisme. Il s'agit donc de savoir si le fait d'appartenir à une composante donnée de la population, en l'occurrence la composante francophone du Nord de l'Ontario, vivant en situation minoritaire, peut expliquer en partie le statut de sansabri absolu ou de personne à risque de le devenir. Dans la mesure où les Francophones minoritaires auraient des caractéristiques et des raisons d'itinérance spécifiques qui augmenteraient chez eux les probabilités d'être ou de devenir sans-abri, la solution à leur situation dépendrait de leur spécificité culturelle ou linguistique. Dès lors, cette étude poursuit les objectifs suivants :

1. Recenser dans les villes de Timmins et de North Bay les sansabri francophones et les Francophones à risque de devenir sans-abri;

2. Décrire les raisons conduisant à leur itinérance;

3. Vérifier s'il existe une association entre l'appartenance à un groupe de la population d'étude et le statut de sans-abri;

4. Comparer les caractéristiques des Francophones avec celles des deux autres composantes de la population d'étude, les Anglophones et les Autochtones; 
5. Comparer les raisons données expliquant le sans-abrisme chez ces trois groupes de la population d'étude.

\section{Méthodologie}

Les données collectées pour le projet Pauvreté, sans-abrisme et migration (PSAM) mené dans le Nord de l'Ontario par la $\mathrm{D}^{\text {re }}$ Carol Kauppi et ses collaborateurs en 2011 ont servi à la présente étude. ${ }^{4}$ Ce projet a la particularité d'étudier la question d'itinérance à travers une intense collaboration entre un réseau local de pourvoyeurs de services, d'actuels et d'anciens sans-abri, de planificateurs et de décideurs politiques, d'étudiants et d'étudiantes ainsi que de chercheurs et de chercheuses universitaires.

\section{Population d'étude}

Le projet PSAM porte sur les populations du nord-est de l'Ontario, spécialement celles de Cochrane, de Moosonee, de Sudbury, de Timmins, de North Bay, de Hearst, de Fort Albany et de Kashechewan, et ce, sans distinction de sexe, d'origine ethnique ou de classes sociales. Quant à la présente étude, elle porte exclusivement sur les populations de Timmins et de North Bay et elle se limite aux personnes âgées de 13 ans ou plus.

En matière de sans-abrisme, il importe de distinguer entre deux groupes de personnes : les "sans-abri absolus » et les hommes ou femmes qui sont "à risque de le devenir ". Le terme "sansabri » employé seul et le terme "itinérants » renvoient à ces deux groupes. Un sans-abri absolu vit dans la rue, dans un refuge, temporairement chez un membre de sa famille, chez des amis ou dans un milieu impropre à l'habitation humaine (Hwang, et collab., 2001). Une personne à risque de devenir sans-abri vit une situation particulière qui l'invite à l'itinérance, tels une expulsion imminente de son logement, un revenu extrêmement faible, un abus familial ou un problème de santé sans prestation d'assurance (Daly, 1996; Kauppi, Bélanger et Partridge, 2003). 


\section{Collecte de données}

Pour la collecte de données, le projet PSAM a utilisé une méthodologie basée sur les services. Les participants ont été recrutés à travers les agences auprès desquelles ils obtiennent assistance. À Timmins comme à North Bay, les opérations de collecte ont été coordonnées et réalisées par des étudiantes et des étudiants universitaires qui ont reçu une formation dans l'administration de questionnaires et la codification des données d'enquête.

À Timmins, le Conseil d'administration de services sociaux du district de Cochrane et le Timmins Native Friendship Centre et, à North Bay, le Nipissing District Homelessness Partnership ont joué un rôle important dans l'identification et le recrutement des fournisseurs de services aux pauvres et aux sans-abri, tels que les banques alimentaires, les soupes populaires, les centres d'accueil, les centres d'hébergement et les services sociaux et services de santé physique et mentale. Nous avons également eu recours à l'internet utilisant Canada411 et en utilisant des Mots clés comme "services sociaux " et "itinérance ».Au total, 21 organismes, programmes et services ont participé au dénombrement des sans-abri à Timmins et 26 à North Bay. Les pourvoyeurs de services ont aidé l'équipe à recruter des participants sans-abri ou à risque de le devenir. L'équipe, composée surtout d'étudiants et étudiantes universitaires provenant de la localité et représentant les trois groupes de la population, les Autochtones, les Anglophones et les Francophones, a assisté les pourvoyeurs de service dans l'administration des questionnaires et a aidé les participants à les remplir s'ils ne pouvaient pas le faire eux-mêmes.

Dans les deux villes, les données ont été recueillies à l'aide d'un questionnaire structuré par la méthode de service, sans porter atteinte à la vie privée des sans-abri qui y ont recours. Le questionnaire a été conçu pour effectuer un dénombrement valide et sans double compte des sans-abri. Il a été adapté du Automated National Client-Specific Homeless Services Recording System (ANCHoR), un système díinformation visant à appuyer la coordination des services aux sans-abri. Il est conçu pour recueillir des renseignements sociodémographiques fondamentaux sur les 
clients des services, notamment : leurs initiales, leurs premier et deuxième prénoms et leur nom de famille, ainsi que leur date de naissance, leur numéro díassistance sociale, leur sexe, leur appartenance ethnique/linguistique ou leur race, leur situation familiale, et les dates du début et de la fin de leur utilisation des services (Peressini, McDonald et Hulchanski, 1996). Les raisons fournies par les personnes pour expliquer leur sans-abrisme ont aussi été répertoriées.

Les entrevues se sont déroulées en anglais ou en français à l'aide d'un questionnaire rédigé dans l'une ou l'autre des deux langues. Des informations détaillées ont été recueillies sur les caractéristiques sociodémographiques des participantes et participants : style de vie, santé, revenu familial, histoire résidentielle, ethnicité, langues parlées, revenu, état civil, nombre d'enfants, antécédents d'itinérance et histoire migratoire. Ce dernier élément comporte plusieurs aspects autour de la migration elle-même, sa durée, ses motifs ainsi que le mode de vie migrant (Pollio, 1997 dans Kauppi, et collab., 2009). En conséquence, la migration est perçue comme un élément fondamental de l'itinérance : les itinérants étant les personnes qui ont quitté leur communauté d'origine, alors que celles qui n'ont jamais déménagé ou migré sont considérées comme non itinérantes (Kauppi, et collab., 2009). Pour mieux cerner le statut de migrant, nous avons pris en considération deux éléments : le lieu de naissance et le sentiment d'appartenance à la ville actuelle de résidence comme étant «sa ville ».Ainsi, est migrante, toute personne qui n'estimait pas sa ville actuelle de résidence comme étant "sa ville " ou une personne dont la ville de résidence ne correspondait pas à sa ville de naissance. En revanche, une personne non migrante est : soit celle dont la ville de résidence correspondait à sa ville de naissance, soit celle qui estimait que cette ville était la sienne.

Dans les deux villes, la collecte de données a été menée sur une période de sept jours : à Timmins, du 23 au 29 janvier 2011; et à North Bay, six mois plus tard, du 20 au 26 juillet 2011. Les collectes de données à Sudbury avaient lieu soit durant la dernière semaine de janvier, soit durant la dernière semaine de juillet, ce qui a motivé le choix de périodes analogues pour Timmins et North 
Bay. Par ailleurs, la fin du mois est propice à une recrudescence des sans-abri (Peressini, McDonald et Hulchanski, 1996). Toutes les agences avaient un endroit sûr où l'équipe rassemblait et entreposait les questionnaires à la fin des entrevues.

\section{Analyses statistiques}

La codification, la saisie et le traitement des données ont été réalisés par des étudiantes et étudiants à l'Université Laurentienne. Après le contrôle du codage et un examen de cohérence, nous avons analysé les données en utilisant le logiciel SPSS (version 19). Pour les analyses statistiques, nous avons d'abord procédé aux analyses descriptives des participants et participantes selon un certain nombre de caractéristiques sociodémographiques.

Notre échantillon des deux villes totalisait 850 personnes qui s'identifiaient comme sans-abri absolus ou à risque de le devenir. Cependant, pour 25 de ses personnes, nous ne pouvions pas déterminer à partir des autres données qu'elles avaient fournies si elles étaient vraiment sans-abri absolus ou à risque de le devenir. Elles ont alors été exclues. De plus, lorsque le sexe était une variable de l'analyse, nous avons exclu 68 cas en raison de données manquantes, ne considérant que les 782 cas restants. Les répondants étaient des adultes dont certains étaient accompagnés d'enfants. Cette étude porte sur les enfants âgés de 13 ans et plus ${ }^{5}$. Par la suite, nous avons fait des analyses bivariées avec des tests de Khi-carré pour comparer les caractéristiques (variables nominales) des Francophones avec celles des Anglophones et des Autochtones. Les tests de Khi-carré ont par ailleurs permis de comparer les niveaux, les risques et les expériences d'itinérance des Francophones à ceux des deux autres groupes. Les effectifs pour ces différentes analyses varient en raison de données manquantes pour certaines variables considérées.

\section{Résultats}

Le Tableau 1 décrit les populations de Timmins et de North Bay selon divers groupes et selon le nombre de sans-abri qui 
y vivent. Pour les deux villes, on observe une forte proportion d'Anglophones (40,1\% à Timmins et $69 \%$ à North Bay) et d'Autochtones (32\% à Timmins et $21 \%$ à North Bay) qui sont à risque de devenir sans-abri. On note aussi une proportion importante d'Autochtones (39,7\% pour Timmins et $29 \%$ pour North Bay) et d'Anglophones (38,2\% pourTimmins et $65 \%$ pour North Bay) de sans-abri absolus. À Timmins, les Francophones représentent environ $26 \%$ des personnes de notre échantillon qui sont à risque de devenir sans-abri et presque $21 \%$ des personnes qui sont des sans-abri absolus, tandis qu'à North Bay, les Francophones représentent $10 \%$ des personnes à risque de devenir sans-abri et $6 \%$ des sans-abri absolus.

En $2006^{6}$, la population générale de Timmins est composée de $46 \%$ d'Anglophones, de $40 \%$ de Francophones, de $8 \%$ d'Autochtones et de $6 \%$ d'allophones, et celle de North Bay de $73 \%$ d'Anglophones, de $16 \%$ de Francophones, de $6 \%$ d'Autochtones et de $5 \%$ d'allophones. Chez les Anglophones, le nombre de sans-abri absolus et de personnes à risque de le devenir correspond approximativement à leur proportion dans la population de Timmins et de North Bay. Or les Francophones sans-abri $(24,4 \%$ et 8,4\%) sont sous-représentés dans l'échantillon par rapport à la population générale de Timmins et de North Bay $(40,1 \%$ et $15,9 \%)$, tandis que les Autochtones sans-abri $(34,2 \%$ et $23,5 \%$ ) sont surreprésentés par rapport à la population générale $(7,7 \%$ et $6,0 \%)$. 
Tableau 1 : Comparaison des personnes âgées de 13 ans et plus en fonction de leur statut de sans-abri par lieu et groupe

\begin{tabular}{|c|c|c|c|c|c|c|c|c|}
\hline \multicolumn{9}{|c|}{ Timmins* } \\
\hline & \multicolumn{2}{|c|}{$\begin{array}{c}\text { Population } \\
2006\end{array}$} & \multicolumn{2}{|c|}{$\begin{array}{c}\text { À risque } \\
2011\end{array}$} & \multicolumn{2}{|c|}{$\begin{array}{c}\text { Sans-abri } \\
\text { absolus } 2011\end{array}$} & \multicolumn{2}{|c|}{$\begin{array}{l}\text { Total } \\
2011\end{array}$} \\
\hline $\begin{array}{l}\text { Principales } \\
\text { composantes }\end{array}$ & Effectifs & $\%$ & Effectifs & $\%$ & Effectifs & $\%$ & Effectifs & $\%$ \\
\hline Anglophones & 19725 & 46,4 & 135 & 40,1 & 50 & 38,2 & 185 & 39,5 \\
\hline Francophones & 17055 & 40,1 & 87 & 25,8 & 27 & 20,6 & 114 & 24,4 \\
\hline Autochtones & 3275 & 7,7 & 108 & 32,0 & 52 & 39,7 & 160 & 34,2 \\
\hline Autres & 2500 & 5,9 & 7 & 2,1 & 2 & 1,5 & 9 & 1,9 \\
\hline Total & 42555 & 100 & 337 & 100 & 131 & 100 & 468 & 100 \\
\hline \multicolumn{9}{|c|}{ North Bay } \\
\hline & \multicolumn{2}{|c|}{$\begin{array}{l}\text { Population } \\
2006\end{array}$} & \multicolumn{2}{|c|}{$\begin{array}{c}\text { À risque } \\
2011\end{array}$} & \multicolumn{2}{|c|}{$\begin{array}{c}\text { Sans-abri } \\
\text { absolus } 2011\end{array}$} & \multicolumn{2}{|c|}{$\begin{array}{l}\text { Total } \\
2011\end{array}$} \\
\hline $\begin{array}{l}\text { Principales } \\
\text { composantes }\end{array}$ & Effectifs & $\%$ & Effectifs & $\%$ & Effectifs & $\%$ & Effectifs & $\%$ \\
\hline Anglophones & 38780 & 72,8 & 173 & 69,2 & 70 & 65,4 & 243 & 68,1 \\
\hline Francophones & 8445 & 15,9 & 24 & 9,6 & 6 & 5,6 & 30 & 8,4 \\
\hline Autochtones & 3205 & 6,0 & 53 & 21,2 & 31 & 29,0 & 84 & 23,5 \\
\hline Autres & 2810 & 5,3 & 0 & 0,0 & 0 & 0,0 & 0 & 0,0 \\
\hline Total & 53240 & 100 & 250 & 100 & 107 & 100 & 357 & 100 \\
\hline
\end{tabular}

* Les données manquantes sont dans une plage acceptable, moins de $10 \%$ des cas pour les variables clefs dans cette étude (Belle, 2002; Bryman et Cramer, 1990).

Les informations présentées au Tableau 2 portent sur la situation des personnes âgées de plus de 13 ans et de moins de 19 ans, en fonction de leur groupe d'appartenance et de leur statut de sans-abrisme. Elles reproduisent la répartition de la population de sans-abri en général. Les jeunes Francophones sont sousreprésentés proportionnellement dans notre échantillon tandis que les jeunes autochtones sont surreprésentés. La situation des jeunes autochtones sans-abri est même pire que celle de l'ensemble des Autochtones sans-abri. 
Tableau 2 : Comparaison des enfants et adolescents de moins de 19 ans` en fonction de leur statut de sans-abri par lieu et composante

\begin{tabular}{|c|c|c|c|c|c|c|}
\hline \multicolumn{7}{|c|}{ Timmins } \\
\hline & \multicolumn{2}{|c|}{$\begin{array}{c}\text { À risque } \\
2011\end{array}$} & \multicolumn{2}{|c|}{$\begin{array}{c}\text { Sans-abri absolus } \\
2011\end{array}$} & \multicolumn{2}{|c|}{$\begin{array}{l}\text { Total } \\
2011\end{array}$} \\
\hline Principales composantes & Effectifs & $\%$ & Effectifs & $\%$ & Effectifs & $\%$ \\
\hline Anglophones & 67 & 30,3 & 32 & 29,9 & 99 & 30,2 \\
\hline Francophones & 44 & 19,9 & 18 & 16,8 & 62 & 18,9 \\
\hline Autochtones & 95 & 43,0 & 55 & 51,4 & 150 & 45,7 \\
\hline Autres & 15 & 6,8 & 2 & 1,9 & 17 & 5,2 \\
\hline Total & 221 & 100 & 107 & 100 & 328 & 100 \\
\hline \multicolumn{7}{|c|}{ North Bay } \\
\hline & \multicolumn{2}{|c|}{$\begin{array}{l}\text { À risque } \\
2011\end{array}$} & \multicolumn{2}{|c|}{$\begin{array}{c}\text { Sans-abri absolus } \\
2011\end{array}$} & \multicolumn{2}{|c|}{$\begin{array}{l}\text { Total } \\
2011\end{array}$} \\
\hline Principales composantes & Effectifs & $\%$ & Effectifs & $\%$ & Effectifs & $\%$ \\
\hline Anglophones & 62 & 60,2 & 30 & 68,2 & 92 & 62,6 \\
\hline Francophones & 12 & 11,7 & 3 & 6,8 & 15 & 10,2 \\
\hline Autochtones & 29 & 28,2 & 11 & 25,0 & 40 & 27,2 \\
\hline Autres & 0 & 0,0 & 0 & 0,0 & 0 & 0,0 \\
\hline Total & 103 & 100 & 44 & 100 & 147 & 100 \\
\hline
\end{tabular}

* Ce tableau porte sur les enfants et adolescents, de la naissance à l'âge de 18 ans inclusivement.

Les distributions selon le sexe et le groupe ethnique retenu donnent les résultats suivants : comparativement aux hommes $(45,8 \%)$, les femmes constituent la majorité de l'échantillon $(54,2 \%)$. Indépendamment du sexe, les Anglophones forment la majorité de notre échantillon de sans-abri, soit $52 \%$; viennent ensuite les Autochtones avec près du tiers $(30 \%)$ et les Francophones, $18 \%$.

Le Tableau 3 fait état des caractéristiques de migration et d'itinérance selon le sexe et les différents groupes de notre population d'étude. Peu importe le groupe d'appartenance, on note une plus forte proportion de non-migrants que de migrants, 
et ce, aussi bien chez les femmes que chez les hommes. La proportion des non-migrants varie entre $68 \%$ et $83 \%$. Pour les femmes, on note une supériorité proportionnelle des Anglophones (83\%), alors que ce sont les hommes francophones qui sont proportionnellement plus nombreux parmi les non-migrants $(82,7 \%)$. Parmi les migrants, les femmes francophones $(30 \%)$ et les hommes autochtones (32\%) sont proportionnellement plus nombreux que les autres.

Par ailleurs, notre échantillon est composé de plus de personnes à risque de devenir sans-abri que de sans-abri absolus, peu importe le sexe et le groupe d'appartenance. Cependant, il y a proportionnellement peu d'Anglophones sans-abri absolus, hommes $(8 \%)$ ou femmes (12\%). Les sans-abri absolus sont proportionnellement plus élevés chez les femmes francophones $(25 \%)$ que chez les hommes (17\%). Parmi les personnes à risque de devenir des sans-abri, les hommes anglophones (92\%) et les hommes autochtones $(91 \%)$ sont proportionnellement les plus nombreux. De plus, une majorité d'hommes autochtones (65\%) et anglophones (58\%) et une importante proportion de femmes autochtones $(50 \%)$ et anglophones $(41 \%)$ ont déclaré avoir déjà été sans-abri dans leur vie. Les hommes (57\%) et femmes (79\%) francophones ont été proportionnellement plus nombreux que les autres à dire n'avoir jamais été sans-abri dans leur vie. Des proportions non négligeables d'hommes (45\% chez les Autochtones et $35 \%$ chez les Anglophones) et de femmes (32\% chez les Autochtones et $22 \%$ chez les Anglophones) ont dit avoir été sans-abri dans l'année qui a précédé l'entrevue.

Tableau 3 : Caractéristiques sociodémographiques de la population d'étude en $\%$

\begin{tabular}{|c|c|c|c|c|c|c|}
\hline \multirow[b]{2}{*}{ Caractéristiques } & \multicolumn{3}{|c|}{ Femmes $(\mathrm{N}=407)$} & \multicolumn{3}{|c|}{ Hommes $(\mathrm{N}=346)$} \\
\hline & $\begin{array}{c}\text { Anglophones } \\
(\mathrm{N}=211)\end{array}$ & $\begin{array}{l}\text { Francophones } \\
\qquad(\mathrm{N}=67)\end{array}$ & $\begin{array}{c}\text { Autochtones } \\
(\mathrm{N}=129)\end{array}$ & $\begin{array}{l}\text { Anglophones } \\
(\mathrm{N}=182)\end{array}$ & $\begin{array}{c}\text { Francophones } \\
(\mathrm{N}=65)\end{array}$ & $\begin{array}{c}\text { Autochtones } \\
(\mathrm{N}=99)\end{array}$ \\
\hline $\begin{array}{l}\text { Statut par rapport } \\
\text { à la migration }\end{array}$ & & & & 263 & 173 & 320 \\
\hline - Non-migrants & $\begin{array}{l}10,0 \\
83,2\end{array}$ & $\begin{array}{l}30,2 \\
69,8\end{array}$ & $\begin{array}{l}25,1 \\
76,9\end{array}$ & $\begin{array}{l}73,7 \\
73,5\end{array}$ & $\begin{array}{l}11,3 \\
82,7\end{array}$ & $\begin{array}{l}3,0 \\
68,0\end{array}$ \\
\hline
\end{tabular}




\begin{tabular}{|c|c|c|c|c|c|c|}
\hline $\begin{array}{l}\text { Sans-abri } \\
\text { - Absolus } \\
\text { - À risque }\end{array}$ & $\begin{array}{l}11,8 \\
88,2\end{array}$ & $\begin{array}{l}25,4 \\
74,6\end{array}$ & $\begin{array}{l}19,4 \\
80,6\end{array}$ & $\begin{array}{r}8,2 \\
91,8\end{array}$ & $\begin{array}{l}16,9 \\
83,1\end{array}$ & $\begin{array}{r}9,1 \\
90,9\end{array}$ \\
\hline $\begin{array}{l}\text { Ayant déjà été } \\
\text { sans-abri dans } \\
\text { sa vie } \\
\text { - Oui } \\
\text { - Non }\end{array}$ & $\begin{array}{l}40,7 \\
59,3\end{array}$ & $\begin{array}{l}21,4 \\
78,6\end{array}$ & $\begin{array}{l}50,5 \\
49,5\end{array}$ & $\begin{array}{l}57,5 \\
42,5\end{array}$ & $\begin{array}{l}43,4 \\
56,6\end{array}$ & $\begin{array}{l}65,4 \\
34,6\end{array}$ \\
\hline $\begin{array}{l}\text { Ayant été sans- } \\
\text { abri l'an passé } \\
\text { - Oui } \\
\text { - Non }\end{array}$ & $\begin{array}{l}22,1 \\
77,9\end{array}$ & $\begin{array}{l}14,3 \\
85,7\end{array}$ & $\begin{array}{l}32,4 \\
67,6 \\
\end{array}$ & $\begin{array}{l}35,0 \\
65,0 \\
\end{array}$ & $\begin{array}{l}22,6 \\
77,4 \\
\end{array}$ & $\begin{array}{l}44,9 \\
55,1\end{array}$ \\
\hline $\begin{array}{l}\text { Ayant dormi dans } \\
\text { la rue l'an passé } \\
\text { - Oui } \\
\text { - Non }\end{array}$ & $\begin{array}{r}8,2 \\
91,8\end{array}$ & $\begin{array}{r}1,9 \\
98,1\end{array}$ & $\begin{array}{l}10,6 \\
89,4\end{array}$ & $\begin{array}{l}17,1 \\
82,9\end{array}$ & $\begin{array}{r}6,1 \\
93,9\end{array}$ & $\begin{array}{l}27,1 \\
72,9\end{array}$ \\
\hline
\end{tabular}

Le Tableau 4a et le Tableau 4b comparent d'un côté les Francophones aux Anglophones et de l'autre, les Francophones aux Autochtones, selon certaines caractéristiques sociodémographiques et l'expérience d'itinérance et de migration.

Selon les résultats présentés dans le Tableau $4 a$, nous n'avons noté aucune différence significative entre les Francophones et les Anglophones quant à leurs traits sociodémographiques, c'est-àdire leur sexe, leur âge et leur statut matrimonial. Cependant, on note des différences significatives quant aux diverses expériences d'itinérance, plus particulièrement chez les personnes ayant été sans-abri dans leur vie ou au cours de l'année précédant l'entrevue et ayant dormi dans la rue au cours de l'année précédant l'enquête. En effet, les tests de Khi-carré indiquent clairement que les Francophones et les Anglophones ne sont pas comparables sur le plan d'antécédent d'itinérance [ayant déjà été sans-abri dans sa vie $(\mathrm{P}<0,001)$; étaient sans-abri l'an passé $(\mathrm{P}<0,046)$; et ayant dormi dehors ou dans la rue l'an passé $(\mathrm{P}<0,027)]$, avec une situation plus favorable pour les Francophones. 
Selon le Tableau 4b, il n'y a aucune différence significative entre les Francophones et les Autochtones quant à l'âge et au sexe, deux des trois variables sociodémographiques considérées. Toutefois, il y en a une pour le statut matrimonial. Plus d'Autochtones que de Francophones étaient célibataires, tandis que plus de Francophones que d'Autochtones étaient séparés ou divorcés. Afin de comprendre l'importance de cette différence, nous aurions besoin de données qui n'ont pas fait l'objet de notre cueillette lors de cette enquête. Quant aux variables portant sur les expériences antécédentes d'itinérance, les Francophones et les Autochtones n'ont pas le même profil. D'abord, les Autochtones sont beaucoup plus susceptibles d'être sans-abri absolus ou à risque de le devenir, d'avoir été sans-abri au cours de leur vie ou dans l'année précédant l'entrevue, et d'avoir dormi dans la rue au cours de l'année précédant l'entrevue. Cela dit, le statut migratoire des Francophones et celui des Autochtones ne diffèrent pas de façon significative. Des enquêtes plus approfondies sont nécessaires afin de comprendre ce phénomène relevé lors de cette étude.

Tableau 4a : Comparaison de certaines caractéristiques des Francophones avec celles des Anglophones

\begin{tabular}{|c|c|c|c|c|c|}
\hline \multirow{2}{*}{$\begin{array}{l}\text { Caractéristiques sociodémographiques } \\
\text { et d'itinérance }\end{array}$} & \multicolumn{2}{|c|}{ Francophones } & \multicolumn{2}{|c|}{ Anglophones } & \multirow[b]{2}{*}{$\begin{array}{l}\text { P-Values } \\
\text { Khi-carré }\end{array}$} \\
\hline & $N=132$ & $\%$ & $N=393$ & $\%$ & \\
\hline $\begin{array}{l}\text { Sexe } \\
\text { - Hommes } \\
\text { - Femmes }\end{array}$ & $\begin{array}{l}67 \\
65 \\
\end{array}$ & $\begin{array}{l}50,8 \\
49,2 \\
\end{array}$ & $\begin{array}{l}211 \\
182 \\
\end{array}$ & $\begin{array}{l}53,7 \\
46,3\end{array}$ & .599 \\
\hline $\begin{array}{l}\text { Groupes d'âge en années révolues } \\
\text { - } 13-19 \\
\text { - } 20-29 \\
\text { - } 30-39 \\
\text { - } 40-49 \\
\text { - } 50-59 \\
\text { - } 60 \text { et plus }\end{array}$ & $\begin{array}{l}18 \\
24 \\
30 \\
29 \\
26 \\
17\end{array}$ & $\begin{array}{l}12,5 \\
16,7 \\
20,8 \\
20,1 \\
18,1 \\
11,8\end{array}$ & $\begin{array}{l}74 \\
81 \\
72 \\
92 \\
78 \\
31\end{array}$ & $\begin{array}{r}17,3 \\
18,9 \\
16,8 \\
21,5 \\
18,2 \\
7,2\end{array}$ & .350 \\
\hline
\end{tabular}




\begin{tabular}{|c|c|c|c|c|c|}
\hline $\begin{array}{l}\text { Statut matrimonial } \\
\text { - Mariés/union de fait } \\
\text { - Célibataire } \\
\text { - Séparé ou divorcé } \\
\text { - Veuf(ve) }\end{array}$ & $\begin{array}{r}46 \\
52 \\
33 \\
4\end{array}$ & $\begin{array}{r}34,1 \\
38,5 \\
24,4 \\
3,1\end{array}$ & $\begin{array}{r}125 \\
183 \\
72 \\
12\end{array}$ & $\begin{array}{r}31,9 \\
46,7 \\
18,4 \\
3,1\end{array}$ & .319 \\
\hline $\begin{array}{l}\text { Sans-abri } \\
\text { - Absolus } \\
\text { - À risque }\end{array}$ & $\begin{array}{r}33 \\
111 \\
\end{array}$ & $\begin{array}{l}22,9 \\
77,1\end{array}$ & $\begin{array}{l}120 \\
308\end{array}$ & $\begin{array}{l}28,0 \\
72,0\end{array}$ & .230 \\
\hline $\begin{array}{l}\text { Ayant déjà été sans-abri dans sa vie } \\
\text { - Oui } \\
\text { - Non }\end{array}$ & $\begin{array}{l}39 \\
82\end{array}$ & $\begin{array}{l}32,2 \\
67,8\end{array}$ & $\begin{array}{l}183 \\
187\end{array}$ & $\begin{array}{l}49,5 \\
50,5\end{array}$ & $<.001$ \\
\hline $\begin{array}{l}\text { Étaient sans-abri l'an passé } \\
\text { - Oui } \\
\text { - Non }\end{array}$ & $\begin{array}{l}23 \\
98 \\
\end{array}$ & $\begin{array}{l}19,0 \\
81,0 \\
\end{array}$ & $\begin{array}{l}102 \\
260\end{array}$ & $\begin{array}{l}28,2 \\
71,8 \\
\end{array}$ & .046 \\
\hline $\begin{array}{l}\text { Ayant dormi dehors ou dans la rue l'an } \\
\text { passé } \\
\text { - Oui } \\
\text { - Non }\end{array}$ & $\begin{array}{r}5 \\
106 \\
\end{array}$ & $\begin{array}{r}4,5 \\
95,5 \\
\end{array}$ & $\begin{array}{r}39 \\
292 \\
\end{array}$ & $\begin{array}{l}11,8 \\
88,2 \\
\end{array}$ & .027 \\
\hline $\begin{array}{l}\text { Statut par rapport à la migration } \\
\text { - Migrants } \\
\text { - Non-migrants }\end{array}$ & $\begin{array}{l}29 \\
88\end{array}$ & $\begin{array}{l}24,8 \\
75,2\end{array}$ & $\begin{array}{r}71 \\
283\end{array}$ & $\begin{array}{l}20,1 \\
79,9\end{array}$ & .278 \\
\hline
\end{tabular}


Tableau 4b : Comparaison de certaines caractéristiques des Francophones avec celles des Autochtones

\begin{tabular}{|c|c|c|c|c|c|}
\hline \multirow{2}{*}{$\begin{array}{l}\text { Caractéristiques sociodémographiques } \\
\text { et d'itinérance }\end{array}$} & \multicolumn{2}{|c|}{ Francophones } & \multicolumn{2}{|c|}{ Anglophones } & \multirow[b]{2}{*}{$\begin{array}{l}\text { P-Values } \\
\text { Khi-carré }\end{array}$} \\
\hline & $N=132$ & $\%$ & $N=228$ & $\%$ & \\
\hline $\begin{array}{l}\text { Sexe } \\
\text { - Hommes } \\
\text { - Femmes }\end{array}$ & $\begin{array}{l}65 \\
67\end{array}$ & $\begin{array}{l}49,2 \\
50,8\end{array}$ & $\begin{array}{r}99 \\
129\end{array}$ & $\begin{array}{l}43,4 \\
56,6\end{array}$ & .285 \\
\hline $\begin{array}{l}\text { Groupes d'âge en années révolues } \\
\text { - } 13-19 \\
\text { - } 20-29 \\
\text { - } 30-39 \\
\text { - } 40-49 \\
\text { - } 50-59 \\
\text { - } 60 \text { et plus } \\
\end{array}$ & $\begin{array}{l}18 \\
24 \\
30 \\
29 \\
26 \\
17\end{array}$ & $\begin{array}{l}12,5 \\
16,7 \\
20,8 \\
20,1 \\
18,1 \\
11,8\end{array}$ & $\begin{array}{l}48 \\
52 \\
43 \\
45 \\
37 \\
19\end{array}$ & $\begin{array}{r}19,7 \\
21,3 \\
17,6 \\
18,4 \\
15,2 \\
7,8 \\
\end{array}$ & .268 \\
\hline $\begin{array}{l}\text { Statut matrimonial } \\
\text { - Mariés/union de fait } \\
\text { - Célibataire } \\
\text { - Séparé ou divorcé } \\
\text { - Veuf(ve) }\end{array}$ & $\begin{array}{r}46 \\
52 \\
33 \\
4\end{array}$ & $\begin{array}{r}34,1 \\
38,5 \\
24,4 \\
3,0\end{array}$ & $\begin{array}{r}72 \\
123 \\
34 \\
4\end{array}$ & $\begin{array}{r}30,9 \\
52,8 \\
14,6 \\
1,7\end{array}$ & .028 \\
\hline $\begin{array}{l}\text { Sans-abri } \\
\text { - Absolus } \\
\text { - À risque }\end{array}$ & $\begin{array}{r}33 \\
111\end{array}$ & $\begin{array}{l}22,9 \\
77,1\end{array}$ & $\begin{array}{r}83 \\
161 \\
\end{array}$ & $\begin{array}{l}34,0 \\
66,0\end{array}$ & .021 \\
\hline $\begin{array}{l}\text { Ayant déjà été sans-abri dans sa vie } \\
\text { - Oui } \\
\text { - Non }\end{array}$ & $\begin{array}{l}39 \\
82\end{array}$ & $\begin{array}{l}32,2 \\
67,8\end{array}$ & $\begin{array}{r}115 \\
85 \\
\end{array}$ & $\begin{array}{l}57,5 \\
42,5 \\
\end{array}$ & $<.001$ \\
\hline $\begin{array}{l}\text { Étaient sans-abri l'an passé } \\
\text { - Oui } \\
\text { - Non } \\
\end{array}$ & $\begin{array}{l}23 \\
98\end{array}$ & $\begin{array}{l}19,0 \\
81,0\end{array}$ & $\begin{array}{r}75 \\
125 \\
\end{array}$ & $\begin{array}{l}37,5 \\
62,5 \\
\end{array}$ & $<.001$ \\
\hline $\begin{array}{l}\text { Ayant dormi dehors ou dans la rue l'an } \\
\text { passé } \\
\text { - Oui } \\
\text { - Non }\end{array}$ & $\begin{array}{r}5 \\
106\end{array}$ & $\begin{array}{r}4,5 \\
95,5\end{array}$ & $\begin{array}{r}30 \\
158\end{array}$ & $\begin{array}{l}16,0 \\
84,0\end{array}$ & .003 \\
\hline $\begin{array}{l}\text { Statut par rapport à la migration } \\
\text { - Migrants } \\
\text { - Non-migrants }\end{array}$ & $\begin{array}{l}29 \\
88\end{array}$ & $\begin{array}{l}24,8 \\
75,2\end{array}$ & $\begin{array}{r}52 \\
141 \\
\end{array}$ & $\begin{array}{l}26,9 \\
73,1\end{array}$ & .675 \\
\hline
\end{tabular}


En considérant les facteurs mis de l'avant par les répondantes et les répondants pour expliquer leur statut de sans-abri (Tableau 5), nous devons tenir compte du fait qu'une forte proportion (environ $60 \%$ des personnes dans notre échantillon n'a aucun revenu si ce n'est qu'un appui financier provenant de programmes sociaux. Le total de réponses inscrites au Tableau 5 dépasse le nombre de répondantes et de répondants en raison de la possibilité de choix multiples qui leur était offerte parmi les raisons sous-jacentes à leur statut de sans-abri.

Quel que soit le groupe, sans-abri absolus ou à risque de le devenir, les participantes et participants placent en tête de liste les mêmes deux raisons pour expliquer leur état : l'assistance sociale, puis le manque ou la recherche d'emploi. Par la suite, les réponses different et permettent de distinguer nettement les Francophones des deux autres groupes. Ainsi, les Francophones sans-abri absolus et ceux à risque de le devenir mentionnent un faible salaire comme troisième explication à leur statut de sansabri. De leur côté, les Anglophones et les Autochtones qui sont sans-abri absolus invoquent des problèmes de logement, alors que les personnes à risque mettent de l'avant leur situation familiale, y compris les sévices et la violence. Les problèmes de logement apparaissent aussi en troisième lieu chez les Autochtones. Comme quatrième explication à leur situation, les sans-abri absolus font valoir les difficultés familiales, y compris les sévices et la violence. Les Francophones et les Anglophones à risque indiquent aussi en quatrième lieu les problèmes de logement.

Tableau 5 : Raisons avancées pour expliquer le statut de sans-abri`

\begin{tabular}{|l|c|c|c|c|c|c|}
\hline \multirow{2}{*}{ Raisons } & \multicolumn{3}{|c|}{ Absolus } & \multicolumn{3}{c|}{ À risque } \\
\cline { 2 - 7 } & $\begin{array}{c}\text { Anglo- } \\
\text { phones }\end{array}$ & $\begin{array}{c}\text { Franco- } \\
\text { phones }\end{array}$ & $\begin{array}{c}\text { Autoch- } \\
\text { tones }\end{array}$ & $\begin{array}{c}\text { Anglo- } \\
\text { phones }\end{array}$ & $\begin{array}{c}\text { Franco- } \\
\text { phones }\end{array}$ & $\begin{array}{c}\text { Autoch- } \\
\text { tones }\end{array}$ \\
\hline $\begin{array}{l}\text { Manque ou recherche } \\
\text { d'emploi }\end{array}$ & $107(2)$ & $23(2)$ & $75(2)$ & $258(2)$ & $70(2)$ & $160(2)$ \\
\hline Faible salaire & $36(6)$ & $14(3)$ & $22(6)$ & $108(5)$ & $37(3)$ & $50(5)$ \\
\hline Problèmes de logement & $87(3)$ & $9(5)$ & $51(3)$ & $145(4)$ & $29(4)$ & $85(3)$ \\
\hline
\end{tabular}




\begin{tabular}{|l|c|c|c|c|c|c|}
\hline $\begin{array}{l}\text { Difficultés familiales et } \\
\text { divorce }\end{array}$ & $68(4)$ & $10(4)$ & $38(4)$ & $159(3)$ & $29(4)$ & $85(3)$ \\
\hline Assistance sociale & $132(1)$ & $23(1)$ & $98(1)$ & $311(1)$ & $80(1)$ & $207(1)$ \\
\hline $\begin{array}{l}\text { Dépendances } \\
\text { psychotropiques et } \\
\text { alcoolisme }\end{array}$ & $43(5)$ & 3 & $30(5)$ & 75 & $11(6)$ & $50(5)$ \\
\hline $\begin{array}{l}\text { Maladies mentales ou } \\
\text { physiques }\end{array}$ & 32 & $6(6)$ & 16 & $104(6)$ & 25 & 39 \\
\hline Sortie de prison & 23 & 3 & 18 & 37 & 5 & 36 \\
\hline Migration & 19 & 1 & 10 & 37 & 11 & 21 \\
\hline Autres & 6 & 1 & 6 & 17 & 2 & 12 \\
\hline
\end{tabular}

* Le rang des six raisons les plus importantes apparaît entre parenthèses après le nombre total de réponses pour la raison.

\section{Discussion}

La présente étude sur le sans-abrisme dans deux villes du nordest de l'Ontario, comparant les résultats obtenus avec ceux d'une troisième, soit Sudbury, est la première du genre. Elle porte sur 850 personnes âgées de 13 ans et plus de North Bay et de Timmins qui sont sans-abri absolus ou à risque de le devenir. Près du quart des répondants sont Francophones, un peu plus de la moitié sont Anglophones et un tiers sont Autochtones. De ce nombre, 236 personnes sont qualifiées de sans-abri absolus. Au chapitre de l'itinérance, notre étude montre que les Francophones different des Anglophones et des Autochtones. Nos résultats indiquent que les Francophones se distinguent des Autochtones par rapport à l'état matrimonial (séparés ou divorcés), que les femmes francophones sont proportionnellement plus nombreuses que les hommes francophones à être migrantes, que les jeunes Francophones de moins de 19 ans sont sous-représentés dans les catégories de sans-abri absolus ou à risque de le devenir, que les Francophones, hommes et femmes, sont plus nombreux à dire n'avoir jamais été sans-abri auparavant et finalement, que les Francophones 
"D'abord, la situation de sans-abri absolu ou à risque de le devenir semble chez les Francophones de nature plus circonstancielle, liée à une désinsertion familiale, rupture, séparation ou divorce. Les femmes semblent en être plus particulièrement touchées. » expliquent par un faible salaire leur présente situation de sans-abri, absolus ou à risque de le devenir.

Ces données nous amènent à formuler certaines hypothèses à être confirmées lors d'une prochaine enquête. D'abord, la situation de sans-abri absolu ou à risque de le devenir semble chez les Francophones de nature plus circonstancielle, liée à une désinsertion familiale, rupture, séparation ou divorce. Les femmes semblent en être plus particulièrement touchées. Le nombre peu élevé de jeunes Francophones sans-abri absolus ou à risque de le devenir laisse à supposer que les femmes sont comparativement plus avancées en âge. Il faut alors se demander pourquoi les femmes sans-abri sont plus âgées et si la perte du revenu familial se fait sentir plus fortement chez elles.

Notre étude relève quelques similitudes entre les Francophones et les Anglophones et entre les Francophones et les Autochtones sur un certain nombre de caractéristiques sociodémographiques et d'itinérance. Les caractéristiques sur lesquelles il y a ressemblance entre les Francophones et les Anglophones et entre les Francophones et les Autochtones sont l'âge, le sexe, l'état matrimonial (uniquement pour les Anglophones) et le statut de migration. Cependant selon nos résultats, une plus grande proportion d'Autochtones est célibataire tandis qu'un plus grand nombre de Francophones sont séparés ou divorcés.

En général, ces résultats confirment les tendances documentées dans les neuf études précédentes réalisées à Sudbury (Kauppi, et collab., 2009) au chapitre du sexe, de l'âge, des origines de la population à l'étude et de la situation familiale. Dans tous les cas, les Francophones sont sous-représentés parmi les personnes sans-abri absolus ou à risque de le devenir et les Autochtones sont largement surreprésentés.

\section{Le sans-abrisme chez les Francophones}

Cette étude visait à comprendre les similitudes et les différences dans la prévalence du sans-abrisme ainsi que ses effets dans les divers groupes retenus de la population de North Bay et de Timmins. En comparant nos résultats avec ceux de Sudbury 
"Il semble que les sans-abri francophones aient été constamment sous-représentés dans les échantillons par rapport à la population générale de chacune des villes. »

"En dépit du fait que les sans-abri francophones cèdent rapidement aux demandes linguistiques des fournisseurs de services anglophones, nos données montrent qu'ils sont fiers de leur langue et souhaitent être servis en français. "
(Kauppi, et collab., 2009), nous constatons une sous-représentation des Francophones dans les trois villes. Chez les Francophones de Timmins qui représentent $40 \%$ de la population, les sansabri absolus ou à risque de le devenir ne constituent que le quart environ de l'échantillon des sans-abri. À North Bay les Francophones représentent $16 \%$ de la population et les sans-abri absolus ou à risque de le devenir $10 \%$ de l'échantillon. Enfin, les Francophones de Sudbury représentent $29 \%$ de la population et les sans-abri absolus ou à risque de le devenir occupent à peu près $14 \%$ de l'échantillon.

Nous nous sommes alors demandé si les Francophones étaient effectivement sous-représentés parmi les sans-abri ou si, dans la collecte des données, ils étaient inclus avec les Anglophones en se faisant passer pour tels. Il semble que les sans-abri francophones aient été constamment sous-représentés dans les échantillons par rapport à la population générale de chacune des villes. Malgré la collecte de données sur la langue et la composante de la population, les différentes études ont toujours abouti à ce même résultat, soit la sous-représentation des Francophones (Kauppi, Bélanger et Partridge, 2003; Kauppi, et Gasparini, 2007; Kauppi, et collab., 2009).

Quant à la prestation de services en français, même si elle ne faisait pas l'objet de nos enquêtes à Timmins et à North Bay, nos études antérieures à Sudbury permettent d'en arriver à certaines constatations. Nous avons demandé aux sans-abri francophones de Sudbury s'ils s'étaient heurtés à des difficultés linguistiques. Certains ont dit qu'ils faisaient tout ce qui était possible pour se faire servir en français. Cependant, la plupart s'expriment en anglais pour se faire servir plus rapidement ou pour se plier à ce qu'ils perçoivent comme des exigences du système. En dépit $\mathrm{du}$ fait que les sans-abri francophones cèdent rapidement aux demandes linguistiques des fournisseurs de services anglophones, nos données montrent qu'ils sont fiers de leur langue et souhaitent être servis en français. D'ailleurs, nous avons constaté que l'incapacité de certains fournisseurs de services de lire et de parler en français constituait un obstacle pour les sans-abri qui désiraient se prévaloir de services. Des sans-abri dont les documents étaient 
rédigés en français ont dit se voir refuser l'accès à des services quand des fournisseurs anglophones ne pouvaient pas vérifier leur admissibilité parce qu'ils ne comprenaient pas la langue.

Potter et Gauvreau (2005) ont souligné d'autres facteurs. À Ottawa, les Francophones sont sous-représentés parmi les familles et les femmes adultes qui sont sans-abri parce que pour de nombreux nouveaux arrivants par exemple, le français constitue une langue seconde. Par conséquent, ils échapperaient au dénombrement portant uniquement sur la première langue des répondants. La sous-estimation des sans-abri francophones peut aussi provenir de problèmes d'ordre méthodologique et des difficultés associées à la définition de l'itinérance puisque la définition choisie ne sera pas sans effet sur l'estimation du nombre d'itinérants dans un espace donné (Fournier, et Mercier, 1996). Ainsi, contrairement à la collecte de données indirectes, la collecte des données directes sur les sans-abri nécessite du personnel expert et des moyens beaucoup plus importants. Elle requiert une plus grande disponibilité de la part des chercheurs, une expertise de terrain plus étendue et une sensibilité plus marquée dans les interactions :

Cette option comporte des difficultés, notamment la sélection des contacts et, par conséquent, des personnes elles-mêmes. Ainsi, les personnes rencontrées dans le cadre d'un établissement présentent certaines caractéristiques qui ne sont pas nécessairement partagées par l'ensemble ou par des segments importants de la population itinérante. Le chercheur doit donc avoir à l'esprit qu'il ne sélectionne pas un échantillon de la population itinérante, mais un échantillon de personnes utilisant un service donné, répondant à un besoin particulier, à un moment précis. (Laberge, et Roy, 1994, p. 103)

C'est donc dire que la clientèle que l'on y rencontre est sélectionnée sur une base implicite ou, du moins, sur laquelle le chercheur a peu de contrôle. Loin de constituer un potentiel 
"Dans l'ensemble des données de recensement des sans-abri, moins de Francophones étaient sans-abri absolus, mais un plus grand nombre étaient à risque de le devenir. » biais de sélection, cette clientèle convient puisque la collecte de données dans des agences permet d'identifier plus de $90 \%$ des sans-abri vivant en milieu urbain (Peressini, McDonald et Hulchanski, 2010).

Dans l'ensemble des données de recensement des sansabri, moins de Francophones étaient sans-abri absolus, mais un plus grand nombre étaient à risque de le devenir. De plus, comparativement aux Anglophones et aux Autochtones, moins de Francophones étaient sans-abri ou avaient dormi dans la rue au cours de la dernière année. Chacune de ces variables affiche une différence statistique significative entre les trois groupes. Les Francophones de l'échantillon s'en tirent mieux alors que les Autochtones connaissent le plus mauvais sort. Cependant, il n'y a aucune différence dans la proportion des migrants sans-abri de l'échantillon, la proportion étant sensiblement la même pour les trois groupes à l'étude.

\section{Le sans-abrisme chez les Autochtones}

Une étude du sans-abrisme dans les minorités francophones du Nord de l'Ontario invite à se pencher aussi sur le sort d'un autre groupe minoritaire important de la région, bien que moins nombreux, les Autochtones. Notre étude met en lumière leur surreprésentation dans l'échantillon des sans-abri. En effet à Timmins où ils représentent 7,7\% de la population, les Autochtones comptent pour près de $33 \%$ des sans-abri de l'échantillon. À North Bay, les Autochtones représentent $6 \%$ de la population. Or, $25 \%$ des sans-abri de l'échantillon appartiennent à cette minorité.

De surcroit, il se peut que bon nombre d'Autochtones ne recourent pas aux ressources offertes à l'ensemble de la population. Conséquemment, notre étude sous-estime peut-être leur nombre de sans-abri. Lors des entrevues, des répondants ont mentionné que les Autochtones n'utilisent pas les services, et ce, pour diverses raisons, dont leur absence de pertinence culturelle ou le racisme qu'ils y ressentent. On dit aussi que certains Autochtones d'âge mûr évitent les services en raison de certains de leurs aspects, 
comme les symboles religieux qui leur rappellent amèrement les pensionnats. Mais en dépit des difficultés reliées à leur recensement, il est troublant de constater que $51 \%$ des Autochtones de notre échantillon qui sont âgés de moins de 19 ans sont des sans-abri absolus et que $43 \%$ sont à risque de le devenir. Notre société a une responsabilité d'assurer que les besoins variés d'enfants et d'adolescents autochtones soient pris en compte par un accès propice aux ressources sociales. La surreprésentation des sans-abris autochtones dans la société canadienne résulte des désavantages systémiques auxquels ils sont confrontés.

\section{Conclusion}

"Ainsi, nous

encourageons la réalisation d'études qui intègrent et approfondissent des dimensions macrosociétales et l'environnement dans l'analyse $d u$ phénomène de l'itinérance." "
Notre étude a identifié comme principales raisons d'itinérance le manque ou la recherche d'emploi, l'insuffisance salariale et les difficultés de logement. Cela corrobore les conclusions de la revue de la littérature réalisée sur le sujet par Hébert (1999) selon lesquelles plusieurs auteurs expliquent l'itinérance par le contexte économique, les transformations du système économique et leurs effets sur le marché du travail. Les raisons évoquées sont le chômage chronique et de longue durée, l'effritement du modèle salarial, la disparition massive d'emplois manuels ou requérant peu ou pas de compétences, la crise de l'emploi et le fait que nos sociétés exigent de plus en plus de ses membres pour leur permettre d'accéder à l'existence sociale et au marché de l'emploi.

Notre étude est basée sur les données individuelles, mais il aurait été intéressant qu'elle prenne en compte divers aspects macrosociétaux. Ainsi, nous encourageons la réalisation d'études qui intègrent et approfondissent des dimensions macrosociétales et l'environnement dans l'analyse du phénomène de l'itinérance. Cela est d'autant plus important que la participation communautaire est essentielle dans tout travail sur l'itinérance, sa conceptualisation et sa mesure (Frankish, Hwang et Quantz, 2005) et les villes - comme d'ailleurs les populations — ne fournissent pas les mêmes efforts dans la lutte contre l'itinérance. Le PSAM comblerait une partie de ce vide en menant des études écologiques où il combinerait ses 
données avec celles issues notamment du recensement et où, par exemple, des analyses multiniveaux pourraient être appliquées. De telles recherches devraient aussi comparer les différentes politiques de chaque palier gouvernemental et leur impact sur le sans-abrisme et l'itinérance afin d'en avoir un aperçu global (Glasser, Fournier et Costopoulos, 1999; Eberle, Kraus et Serge, 2001). L'itinérance étant la résultante d'une complexe interaction de facteurs sur le plan individuel et sociétal (Herman, Susser et Struening, 1997; Schwartz et Carpenter, 1999), on sortirait des sentiers battus dans ce domaine. La recherche sur l'itinérance s'est trop souvent limitée aux facteurs de risque individuels en mettant l'accent sur la marginalisation ou l'exclusion (Frankish, Hwang et Quantz, 2005).

En dépit de son originalité, notre étude comporte quelques limites. L'une d'elles est d'avoir comparé les données de Timmins avec celles de North Bay alors qu'elles ont été collectées à six mois d'intervalle. Un tel décalage pourrait avoir entraîné une différence de classification en termes d'âge et de statut de sans-abri absolu ou à risque de le devenir. Nous estimons cependant que six mois de décalage ne devraient pas entraîner de grandes différences ou erreurs de classification au point de modifier nos conclusions. Les enquêtes antérieures à Sudbury avaient été conduites aussi à des intervalles de six mois, de janvier à juillet 2003. Les résultats de ces études avaient une ressemblance remarquable. Ni la saison, ni le laps de temps n'avaient un effet sur les données recueillies.

Une autre limite tient au fait que les données aient été collectées tantôt par des étudiants, tantôt par des agences. On pouvait donc craindre des biais liés notamment à la personnalité des enquêteurs. Une analyse de sensibilité nous aurait permis de nous rendre compte de l'impact de l'administration des questionnaires par des personnes de différents statuts, mais il nous était impossible de distinguer les questionnaires remplis par des agences de ceux remplis par des étudiants. Cependant, le fait que tous les enquêteurs aient suivi une formation dans la conduite des enquêtes et le fait que tous les questionnaires remplis étaient systématiquement vérifiés afin de détecter d'éventuelles aberrations nous rassurent sur la robustesse des données recueillies. 


\section{Notes}

1 Veuillez faire parvenir toute correspondance à Carol Kauppi,École de service social, « Pauvreté, sans-abrisme et migration ", Université Laurentienne, 935, chemin du lac Ramsey Sudbury (Ontario), Canada P3E 2C6. Téléphone : 705-675-1151, poste 5155, téléc. : 705-671-3832. CKauppi@Laurentian.ca

2 Cette étude a été rendue possible grâce au financement obtenu par Carol Kauppi du Conseil de recherche en sciences humaines du Canada, à qui nous exprimons notre reconnaissance, dans le cadre de son programme ARUC, Alliances de recherche universités-communautés. Nous remercions aussi tout le personnel du projet Pauvreté, sans-abrisme et migration pour leur contribution à la collecte de données.

3 Dans cet article, les termes "sans-abrisme " et "itinérance " sont utilisés de manière interchangeable.

4 Avant la réalisation de cette étude, une demande d'approbation éthique a été préparée, soumise et obtenue auprès du Comité d'éthique sur la recherche de l'Université Laurentienne. Les partenaires locaux, soumis à une obligation semblable, devaient obtenir également une approbation éthique auprès de leur propre comité d'éthique.

5 Les données manquantes sont dans une plage acceptable, moins de $10 \%$ des cas pour les variables clefs dans cette étude (Belle, 2002; Bryman, et Cramer, 1990).

6 Au moment de la rédaction de ce texte, Statistique Canada avait annoncé les chiffres de population du Recensement de 2011, mais non les profils des communautés. Nous avons alors utilisé les données du Recensement de 2006 et les profils des communautés provenant de ce recensement.

\section{Bibliographie}

BEGIN, Patricia, et collab. (1999). Homelessness, Ottawa, Parliamentary Research Branch, Ottawa, $20 \mathrm{p}$.

BELLE, Gerarld van (2002). Statistical Rules of Thumb, New York, Wiley-Interscience, 248 p.

BOUCHARD, Louise, et collab. (2009). "La santé en situation linguistique minoritaire ", Healthcare Policy, Vol. 4, No 4, p. 33-40.

BRYMAN, Alan, et Duncan CRAMER (1990). Quantitative data analysis for social scientists, London, Routledge, $290 \mathrm{p}$.

CITY OF TORONTO (2000). «The Toronto report card on homelessness 2000 », Toronto, City of Toronto, Ontario.

DALY, Gerald (1996). Homeless : policies, strategies and lives on the street, New York, Routledge, $312 \mathrm{p}$.

EBERLE, Margaret, Deborah KRAUS et Luba SERGE (2001). "Homelessness : Causes and effects - Background report : A profile and policy review of homelessness in the provinces of Ontario, Quebec and Alberta ", Vol. 4, Vancouver, B.C., Ministry of Social Development and Economic Security.

FOURNIER, Louise, et Céline MERCIER (dirs.) (1996). Sans domicile fixe. Au-delà du stéréotype, Montréal, Les Éditions du Méridien, 341 p. 
FRANKISH, C. James, Stephen W. HWANG et Darryl QUANTZ (2005). "Homelessness and health in Canada : Research lessons and priorities ", Canadian Journal of Public Health, 96, CBCA Reference, p. S23.

GLASSER, Irene, Louise FOURNIER et André COSTOPOULOS (1999). " Homelessness in Quebec City, Quebec and Hartford, Connecticut :A cross-national and cross-cultural analysis ", Urban Anthropology, 28, p. 141-59.

HÉBERT,Alain (1999). «Socioéconomie de l'itinérance. Portrait général sur l'itinérance », Rapport de recherche Volet 1, Collectif de recherche sur l'itinérance, la pauvreté et l'exclusion sociale (CRI), Observatoire montréalais du développement, Université du Québec à Montréal.

HERMAN Daniel, Ezra S. SUSSER et Elmer S. STRUENING (1997). "Adverse childhood experiences : Are they risk factors for adult homelessness? ", American Journal of Public Health, 87 , p. $249-55$.

HWANG, Stephen W., et collab. (2011). «Chronic pain among homeless persons : characteristics, treatment, and barriers to management », BMC Family Practice, réf. d'octobre 2011. http://www. biomedcentral.com/1471-2296/12/73.

KAUPPI, Carol, Jean-Marc BÉLANGER et Cheryle PARTRIDGE (2003). Report on homelessness in Sudbury : Time 7, Comparison of findings July 2000 to July 2003, Sudbury, Social Planning Council of Sudbury.

KAUPPI, Carol, et Janet GASPARINI (2007). Homelessness in Sudbury: A Re-Assessment. 2007 update and comparison with findings from the study of homelessness in Sudbury, July 2000 to July 2003, Sudbury, Social Planning Council of Sudbury.

KAUPPI, Carol, et collab. (2009). Migratory and transient homelessness in Northern Ontario : A study of the pathways to becoming homeless in Sudbury and its related impact, Final Report, Sudbury, Social Planning Council of Sudbury.

LABER GE, Danielle, et Shirley ROY (1994). Interroger l'itinérance : stratégies et débats de recherche, Cahiers de recherche sociologique, $\mathrm{N}^{\circ}$ 22, p. 93-112.

PERESSINI, Tracy, Lynn MCDONALD et David HULCHANSKI (1996). Estimating homelessness : Towards a methodology for counting the homeless in Canada, Ottawa, Société canadienne d'hypothèques et de logement.

PERESSINI, Tracy, Lynn MCDONALD et David HULCHANSKI (2010). «Towards a strategy for counting the homeless ", dans David HULCHANSKI, et collab. (dirs.) Finding Home : Policy Options for Addressing Homelessness in Canada (e-book), Toronto : Cities Centre, University of Toronto, chapitre 8.3. réf. de mai 2012. www.homelesshub.ca/FindingHome.

PICARD, Louise, et Gratien ALLAIRE (2005). Deuxième rapport sur la santé des Francophones de l'Ontario. Sudbury, Division du programme de recherche, d'éducation et de développement en santé publique (REDSP) et Institut franco-ontarien.

PICARD, Louise (2000). Rapport sur la santé des Francophones de l'Ontario, Sudbury, Division du programme de recherche, d'éducation et de développement en santé publique (REDSP).

POTTER, Joyce, et Yvon GAUVREAU (2005). "Les services en français pour les sans-abri », Rapport préparé pour l'Équipe de renforcement de la capacité communautaire afin de résoudre la question des sans-abri d'Ottawa, Ottawa. 
QUANTZ, Darryl, et James C. FRANKISH (2002). "Homelessness research in the Greater Vancouver Regional District : An agenda for moving forward », Vancouver, B.C., University of British Columbia.

SHWARTZ, Sharon, et Kenneth M. CARPENTER (1999). "The right answer for the wrong question : Consequences of type III error for public health research [comment] ", American Journal of Public Health, 89, p. 1175-1180.

STATISTIQUE CANADA (2002). Le Recensement de 2001 : Série "Analyses ». Logements collectifs, Ottawa, Ontario.

STROIK, Sharon (2004). Biennal count of homelessness persons in Calgary : Enumerated in emergency and transitional facilities, by service agencies, and on the Streets - 2004, Calgary, Alberta, City of Calgary.

WOODWARD, Jim, et collab. (2002). Research Project on Homelessness in Greater Vancouver, Vancouver, B.C., Greater Vancouver Regional District. 